\title{
Clinicopathologic factors influencing postoperative prognosis in patients with small-sized adenocarcinoma of the lung
}

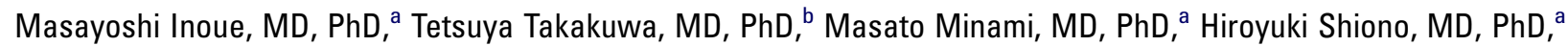
Tomoki Utsumi, MD, PhD, ${ }^{a}$ Yoshihisa Kadota, MD, PhD, ${ }^{a}$ Takuma Nasu, MD, PhD, ${ }^{\mathrm{b}}$ Katsuyuki Aozasa, MD, PhD, ${ }^{\mathrm{b}}$ and Meinoshin Okumura, MD, $\mathrm{PhD}^{\mathrm{a}}$

From the Departments of General Thoracic Surgery $^{\mathrm{a}}$ and Pathology, ${ }^{\mathrm{b}}$ Osaka University Graduate School of Medicine, Osaka, Japan.

Received for publication June 13, 2007; revisions received Sept 3, 2007; accepted for publication Oct 19, 2007.

Address for reprints: Masayoshi Inoue, MD, $\mathrm{PhD}$, Department of General Thoracic Surgery, Osaka University Graduate School of Medicine, 2-2 (L5) Yamadaoka, Suita-city, Osaka 565-0871, Japan (E-mail: mi@ thoracic.med.osaka-u.ac.jp).

J Thorac Cardiovasc Surg 2008;135:830-6 $0022-5223 / \$ 34.00$

Copyright (C) 2008 by The American Association for Thoracic Surgery

doi:10.1016/j.jtcvs.2007.10.034
Objective: Recent technologic developments in computed tomography have increased the incidence of surgical intervention for small-sized lung cancer. Although indications of a sublobar resection for early disease have been discussed, we occasionally encounter locally advanced small-sized lung cancer with node metastasis. The present study aimed to clarify the histopathologic factors influencing nodal involvement and prognosis of such patients.

Methods: We studied 97 patients who underwent complete resection for an adenocarcinoma of $2 \mathrm{~cm}$ or less in diameter. Lymph node metastasis and necrosis were microscopically evaluated, whereas immunohistochemical studies were also performed with Ki-67 and D2-40 for proliferation activity and lymphatic invasion, respectively. In addition, carcinoembryonic antigen expression in the tumor and its level in serum were investigated. Survival analysis was then conducted by using these clinicopathologic factors.

Results: The 5-year disease-free survival rate was $90 \%$. Nodal involvement was significantly frequent in patients with tumors showing microscopic necrosis, a Ki-67 labeling index of greater than 5\%, and an increased serum carcinoembryonic antigen level. Furthermore, 5-year disease-free survival was worse in patients with lesions showing microscopic necrosis (68\%), a Ki-67 labeling index of greater than 5\% (76\%), and lymphatic invasion detected with D2-40 staining (77\%). Multivariate analysis identified lymphatic invasion detected with D2-40 to be an independent predictor for postoperative recurrence.

Conclusions: These results indicate that microscopic necrosis, Ki-67 labeling index, and serum carcinoembryonic antigen level are predictors of nodal involvement. Careful postoperative follow-up examinations for recurrence are required for patients with tumors that show microscopic necrosis, high Ki-67 labeling index, and lymphatic invasion, even in those with stage IA disease.

$\mathrm{W}$ ith the development of high-resolution computed tomography (CT) and its application for cancer screening, small-sized peripheral lung cancer is more frequently encountered. A bronchioloalveolar carcinoma that shows ground-glass opacity (GGO) in CT imaging without vessel or lymphatic spread determined histologically has been recognized as a less-invasive subgroup of small-sized adenocarcinomas. ${ }^{1,2}$ The Noguchi classification, which was first reported in 1995 and is popular in Japan, classifies peripheral adenocarcinomas as those of $2 \mathrm{~cm}$ or less in diameter. ${ }^{3}$ Because of their low-grade malignant feature, several studies have evaluated the results of a lesser pulmonary resection by using preoperative information in regard to GGO area or tumor diameter and found it to be safe on the basis of similar outcomes compared with outcomes in patients who underwent a lobectomy. ${ }^{4-7}$ However, it is well known that lymph node metastasis is not rare in patients with lung 


\section{Abbreviations and Acronyms \\ $\mathrm{CEA}=$ carcinoembryonic antigen \\ $\mathrm{CT}=$ computed tomography \\ GGO $=$ ground-glass opacity \\ LI = labeling index}

cancer with tumors smaller than $2 \mathrm{~cm}$ because the frequency of nodal involvement was reported to be $15 \%$ to $28 \%$ for tumors sized $2 \mathrm{~cm}$ or less and $15 \%$ for solid subcentimeter lesions. ${ }^{8-10}$ Thus it is important to identify patients with locally advanced cancer with nodal metastasis originating from small-sized lung cancer to make proper decisions regarding operative mode and adjuvant treatment.

In the present study we analyzed several potential prognostic factors, including histologic necrosis, Ki-67 labeling index (LI), carcinoembryonic antigen (CEA) expression, serum CEA level, and degree of lymphatic invasion based on the D2-40 antibody for lymphangioendothelium, in patients with a pulmonary adenocarcinoma of $2 \mathrm{~cm}$ or less in diameter. Furthermore, we evaluated the capability of those factors to predict advanced disease with a worse outcome before or during the operation. We believe that the clinicopathologic predictors revealed in the present study can contribute to the improvement of outcome in patients with a small-sized adenocarcinoma who undergo a complete resection.

\section{Materials and Methods \\ Patients}

We retrospectively reviewed the records of 565 patients who underwent thoracic surgery for non-small cell lung cancer between 1992 and 2003 at Osaka University Hospital. Of those, 97 who underwent a complete resection of a peripheral adenocarcinoma of $2 \mathrm{~cm}$ or less in diameter were enrolled, whereas patients treated with induction therapy or with multiple primary lesions were excluded. None were indicated for a mediastinoscopy. The patient characteristics are shown in Table 1. Postoperative follow-up was completed in 90 patients, and the mean follow-up period was 55 months. Prethoracotomy histologic diagnosis was obtained in 69 (70\%) patients: in 43 by using a CT-guided percutaneous needle biopsy, in 25 by using a transbronchial lung biopsy, and in 1 by using sputum cytology. A lobectomy with systematic hilar and mediastinal lymph node dissection was performed in principle. A limited resection was indicated for poor-risk cases, including patients with low pulmonary function, cardiovascular comorbidity, advanced age, and severe diabetes mellitus, as well as for those with a pure GGO lesion smaller than $1.5 \mathrm{~cm}$ in diameter. Postoperative staging was performed according to TNM classification. ${ }^{11}$

\section{Histologic Evaluation}

Informed written consent for research use of the surgical specimens was obtained from all of the patients. Surgically resected specimens were fixed in $10 \%$ formalin and routinely processed for paraffin embedding. Histologic sections were cut into $4-\mu \mathrm{m}$
TABLE 1. Characteristics of 97 patients who underwent complete resection of a small-sized adenocarcinoma (from our database: 1993-2003)

\begin{tabular}{lc}
\hline Age (y) & 38-82; average, 63.1 \\
Sex & 53 \\
Male & 44 \\
Female & \\
Tumor diameter & 15 \\
$\quad<1 \mathrm{~cm}$ & 13 \\
$1-1.5 \mathrm{~cm}$ & 69 \\
$1.5-2 \mathrm{~cm}$ & \\
Mode & 77 \\
Lobectomy & 16 \\
Segmentectomy & 4 \\
Partial resection & \\
p Stage & 82 \\
IA & 4 \\
IB & 2 \\
IIA & 2 \\
IIB & 7 \\
IIIA & \\
\hline
\end{tabular}

slices, stained with hematoxylin and eosin, and reviewed by 2 pathologists (T.T. and T.N.), who evaluated the presence of microscopic necrosis. Immunohistochemical studies of the paraffin sections were also performed by using the Envision+ system (DAKO, Carpinteria, Calif) for Ki-67 and CEA (DAKO, Kyoto, Japan) and a Vectastain ABC kit (Vector Laboratories, Burlingame, Calif) for D2-40. Antigen retrieval was performed by heating the sections after immersion in Target Retrieval Solution, High $\mathrm{pH}$ (DAKO) in a pressure cooker for 15 minutes. In addition, sections were incubated with primary antibodies (monoclonal antibody for Ki-67 [MIB-1] at a concentration of 1:50 for Ki-67, II-7 at 1:75 for CEA, and D2-40 at 1:120; DAKO) and then lightly counterstained with hematoxylin. Immunohistochemistry with the anti-Ki-67 (clone MIB-1) monoclonal antibody was also performed to estimate the proliferative activity of each tumor, with neoplastic epithelial cells showing intranuclear staining for Ki-67 judged to be positive. The number of Ki-67-positive cells among 200 tumor cells was counted, and the percentage was used as the Ki-67 LI. The Ki-67-high group was defined as those with a Ki-67 LI of greater than 5\%, whereas the Ki-67-low group had a Ki-67 $\mathrm{LI}$ at $5 \%$ or lower. CEA expression was graded as negative $(-)$, no staining observed or membrane staining observed in less than $10 \%$ of tumor cells (weakly positive, + ), weak to moderate complete membrane staining observed in more than $10 \%$ of tumor cells (strongly positive, ++ ), and strong complete membrane staining observed in more than $10 \%$ of tumor cells. Lymphatic invasion was determined to be positive when cancer cells were present surrounded by lymphangioendothelium with D2-40 expression.

\section{Statistical Analyses}

Survival probability was calculated by using the Kaplan-Meier method. The prognostic influence of the variables on survival was analyzed by using a Wilcoxon test and Cox regression. A $\chi^{2}$ or Fisher exact test was used to compare ratios between 2 groups. 


\section{Results}

\section{Results of Surgical Intervention}

The 5-year overall and disease-free survival rates were $93 \%$ and $90 \%$, respectively. Lymph node metastasis was detected in $11(11 \%)$ patients, and the pathologic stage was pIA (T1 N0 M0) in 82, IB (T2 N0 M0) in 4, IIA (T1 N1 M0) in 2, IIB (T2 N1 M0) in 2, and IIIA (T1 N2 M0) in 7 (Table 2). The 5-year disease-free survival rate was $95 \%$ for patients without lymph node metastasis, which was better than that $(59 \%)$ for patients with nodal involvement $(P<.001)$. According to tumor diameter, the 5 -year disease-free survival rate was $100 \%$ for patients with a tumor of $1 \mathrm{~cm}$ or less in diameter, $92 \%$ for those with a tumor of 1 to $1.5 \mathrm{~cm}$ in diameter, and $89 \%$ for those with a tumor of 1.5 to $2 \mathrm{~cm}$ in diameter.

\section{Microscopic Necrosis}

Irrespective of the presence or absence of necrosis recognized at a macroscopic level, tumor cell necrosis was present at the microscopic level in $17(18 \%)$ patients (Figure 1, A). Among those patients with necrotic findings in the tumor, 5 (29\%) had lymph node metastasis, whereas only $6(8 \%)$ of the 78 patients without necrotic findings showed nodal involvement $(P=.022$, Table 2$)$. The 5-year disease-free survival for patients with microscopic necrosis in the tumor was $68 \%$, which was significantly worse than that for patients without necrosis $(P<.001$; Figure $2, A)$. Analysis of the 82 patients with stage IA disease without nodal involvement showed a significant difference in regard to disease-free period between those with positive and negative results for necrosis $(P=.035$; Figure 2, $B)$.

\section{Ki-67 LI}

Ki-67 values varied from no staining to more than $30 \%$ positive cells in small-sized adenocarcinomas. Thirty (31\%) patients had a $\mathrm{Ki}-67 \mathrm{LI}$ of greater than $5 \%$ (Ki-67-high group), in whom 15 tumors showed a value of greater than $10 \%$ (Figure $1, B)$. Lymph node metastasis was detected in $8(27 \%)$ of the 30 patients in the Ki-67-high group, which was more frequent compared with that for the Ki-67-low

TABLE 2. Relationships between lymph node metastasis and microscopic necrosis, Ki-67 labeling index, and serum carcinoembryonic antigen level

\begin{tabular}{lccc}
\hline & LN positive & LN negative & $P$ value \\
\hline Necrosis (+) & $5(29 \%)$ & 12 & \\
Necrosis (-) & $6(8 \%)$ & 74 & .022 \\
Ki-67 LI $<5 \%$ & $3(4 \%)$ & 64 & \\
Ki-67 LI $>5 \%$ & $8(27 \%)$ & 22 & .003 \\
Increased CEA level & $6(25 \%)$ & 18 & \\
Normal CEA level & $5(7 \%)$ & 68 & .025 \\
\hline
\end{tabular}

The cutoff level for serum CEA is $5 \mathrm{ng} / \mathrm{mL}$. The Fisher exact test was used for statistical analysis. $L N$, Lymph node, $L I$, labeling index; $C E A$, carcinoembryonic antigen. group $(P=.003$, Table 2$)$. The 5 -year disease-free survival rate for the Ki-67-high group was 76\%, which was significantly worse than that (99\%) for the Ki-67-low group $(P<.001 ;$ Figure 3, A). Even after analyzing patients with stage IA disease without lymph node metastasis, there was a significant difference in disease-free period between the Ki-67-high and Ki-67-low groups $(P=.037$; Figure 3, $B)$.

\section{CEA Expression by Means of Immunohistochemistry and Serum CEA Level}

CEA expression varied from negative to moderately and strongly positive (Figure $1, C$ ). In immunohistochemistry results tumor cells expressed CEA in $72(74 \%)$ of the patients, among whom $22(23 \%)$ had strongly positive $(++)$ results. Lymph node metastasis was detected in 5 (23\%) of 22 patients with CEA $(++)$ results and $6(8 \%)$ of 75 patients with CEA $(-)$ and $(+)$ results $(P=.118)$. Furthermore, patients with an increased serum CEA level had frequent nodal involvement (Table 2). Prethoracotomy increase of serum CEA levels was observed in 24 (25\%) of 97 patients. Among those patients, nodal metastasis was detected in $6(25 \%)$, which was a higher percentage than those with a normal CEA level (Table 2). Of 13 patients with both increased serum CEA levels and strong CEA staining of the tumors, $5(38.5 \%)$ had lymph node metastasis. In addition, analysis of the relationship between CEA expression in the tumor shown by means of immunohistochemistry and preoperative serum CEA level demonstrated a significant correlation (Table 3). Strong CEA expression was found in 13 (54\%) of 24 patients with increased serum CEA levels. Conversely, $13(59 \%)$ of 22 patients with CEA $(++)$ expression had an increase of serum CEA levels. As for disease-free survival, we found no significant difference between CEA expression level in the tumor and serum CEA level.

\section{Lymphatic Invasion Evaluated With D2-40 Staining of Lymphangioendothelium}

Lymphatic invasion was detected in $18(19 \%)$ of the patients by using immunohistochemistry with D2-40 for visualizing lymphatic vessels (Figure 1,D). The 5-year disease-free survival for those with lymphatic invasion was $77 \%$, which was significantly worse than that $(95 \%)$ for patients without lymphatic invasion $(P<.001$; Figure $4, A)$. When analyzing patients with stage IA disease without lymph node involvement, the 5-year disease-free survival for those with lymphatic invasion tended to be poor compared with that for patients without lymphatic invasion $(P=.061$; Figure 4 , $B)$. No significant correlation was found between lymph node metastasis and lymphatic invasion in the present group of patients.

\section{Multivariate Analysis for Prognostic Influence}

Multivariate analysis with microscopic necrosis, Ki-67 LI, lymphatic invasion detected with D2-40, serum CEA level, 

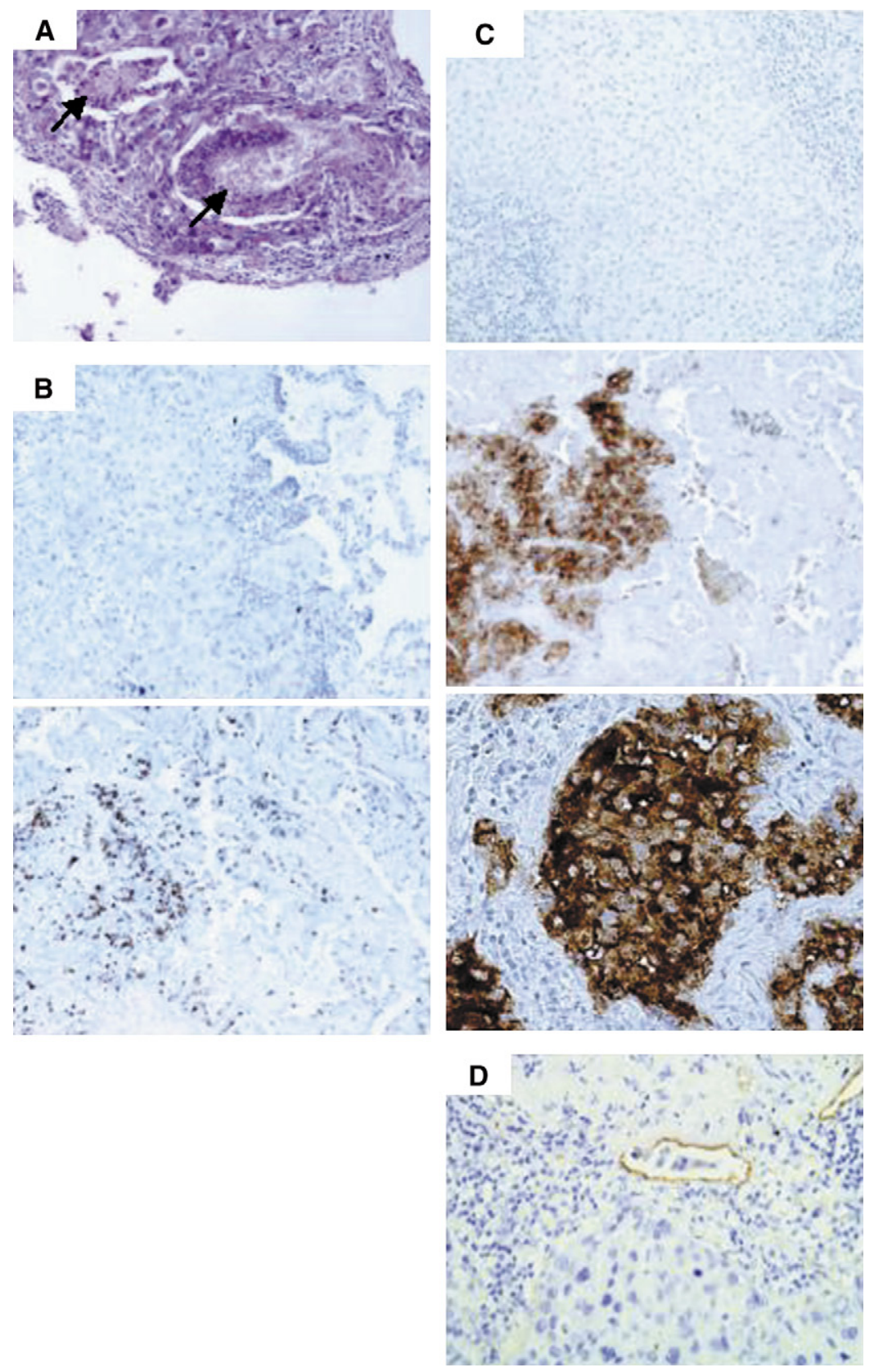

Figure 1. A, Microscopic necrosis (arrow): hematoxylin and eosin staining, original magnification $\times 40$. B, Ki-67 staining: original magnification $\times 40$. Top, Few cancer cells were stained with MIB-1 in this specimen. Immunohistochemistry results classified the tumor as having a Ki-67 labeling index of less than $5 \%$ (Ki-67-low group). Bottom, A proportion of cancer cells was positively stained. Ki-67 labeling index was judged as $\mathbf{2 0} \%$ (Ki-67-high group). C, Carcinoembryonic antigen expression: original magnification $\times \mathbf{4 0}$. Top, Negative staining; middle, moderate expression; bottom, strong expression. D, Immunohistochemistry with D2-40 to evaluate Iymphatic invasion: original magnification $\times \mathbf{4 0}$. Lymphatic invasion by cancer cells was revealed by D2-40 staining of the lymphatic vessels. and p stage as variables identified only lymphatic invasion as an independent predictor for postoperative recurrence (Table 4).

\section{Discussion}

In the present clinicopathologic study, which focused on small-sized pulmonary adenocarcinomas of $2 \mathrm{~cm}$ or less in diameter, we identified the presence of microscopic necrosis, high $\mathrm{Ki}-67 \mathrm{LI}$, and increased serum CEA level as predictors of lymph node metastasis, whereas microscopic necrosis, higher Ki-67 LI, and lymphatic invasion detected with D240 staining were negative factors for disease-free survival. A lesser pulmonary resection for a peripheral small-sized lung cancer with careful node exploration has become an accepted therapy. ${ }^{6,7}$ However, when serum CEA levels are increased in a preoperative examination or if microscopic necrosis is found in preoperative biopsy results or intraoperative rapid diagnosis by using frozen sections, a standard operation with a lobectomy with node dissection should be considered. In addition, patients with a tumor showing 

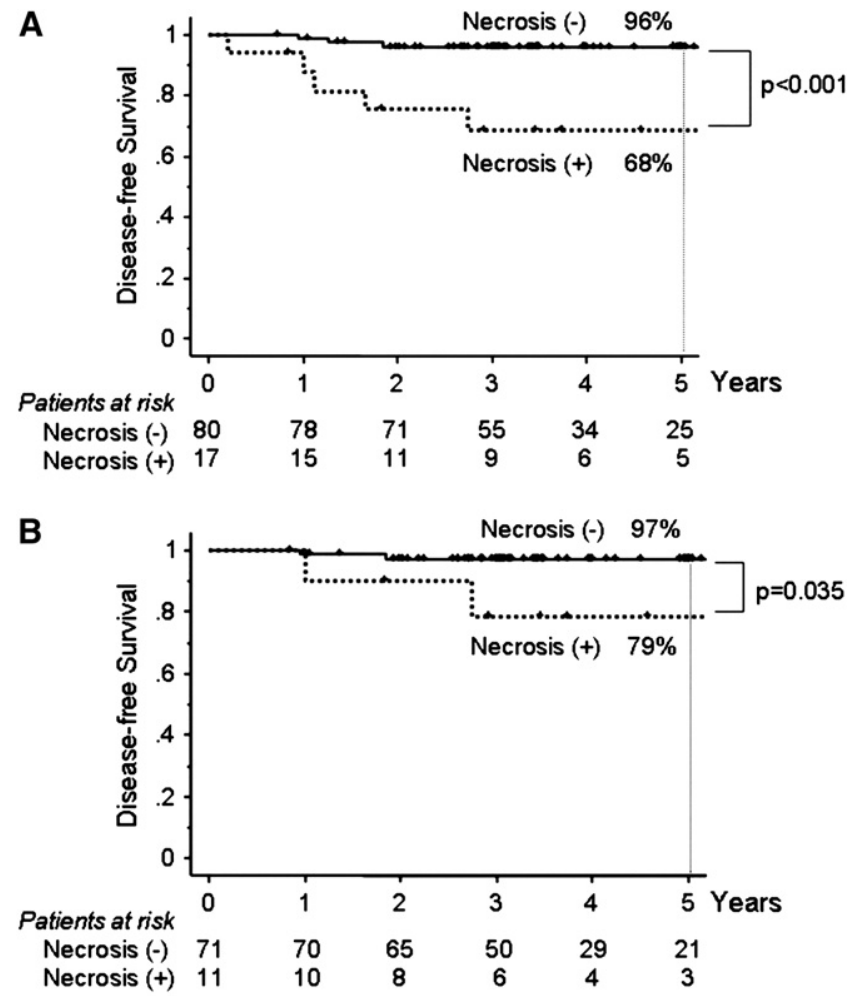

Figure 2. A, Disease-free survival for all patients according to microscopic findings of necrosis. Patients with necrosis in the tumor showed a worse outcome compared with those without necrosis. B, Disease-free survival for patients without nodal metastasis according to microscopic findings of necrosis. The difference in the disease-control ratio was statistically significant.

microscopic necrosis, higher KI-67 LI, or lymphatic invasion might be a candidate for adjuvant therapy, even if the lesion is small and diagnosed as pI.

Kessler and colleagues ${ }^{12}$ reported that a high percentage of necrosis was a negative prognostic factor in addition to blood vessel invasion in patients with resected non-small cell lung carcinomas, although $66 \%$ of those had squamous cell carcinomas. In another report a significant correlation of necrosis with $\mathrm{T}$ stage, tumor size, or invasiveness was documented. ${ }^{13}$ Necrosis is speculated to be caused by chronic cellular hypoxia as a result of rapid tumor progression, and thus it is reasonable that necrotic findings have a negative prognostic effect. In the present study we found a predictive value of microscopic necrosis for nodal involvement and postoperative disease progression in patients with a small-sized adenocarcinoma, in whom macroscopic necrosis is seldom recognized. Furthermore, in our analysis of pIA disease, microscopic necrosis was shown to be a useful prognostic factor for recurrence. Because such microscopic necrosis can be observed intraoperatively in frozen sections and rapidly diagnosed, clinical application is expected.
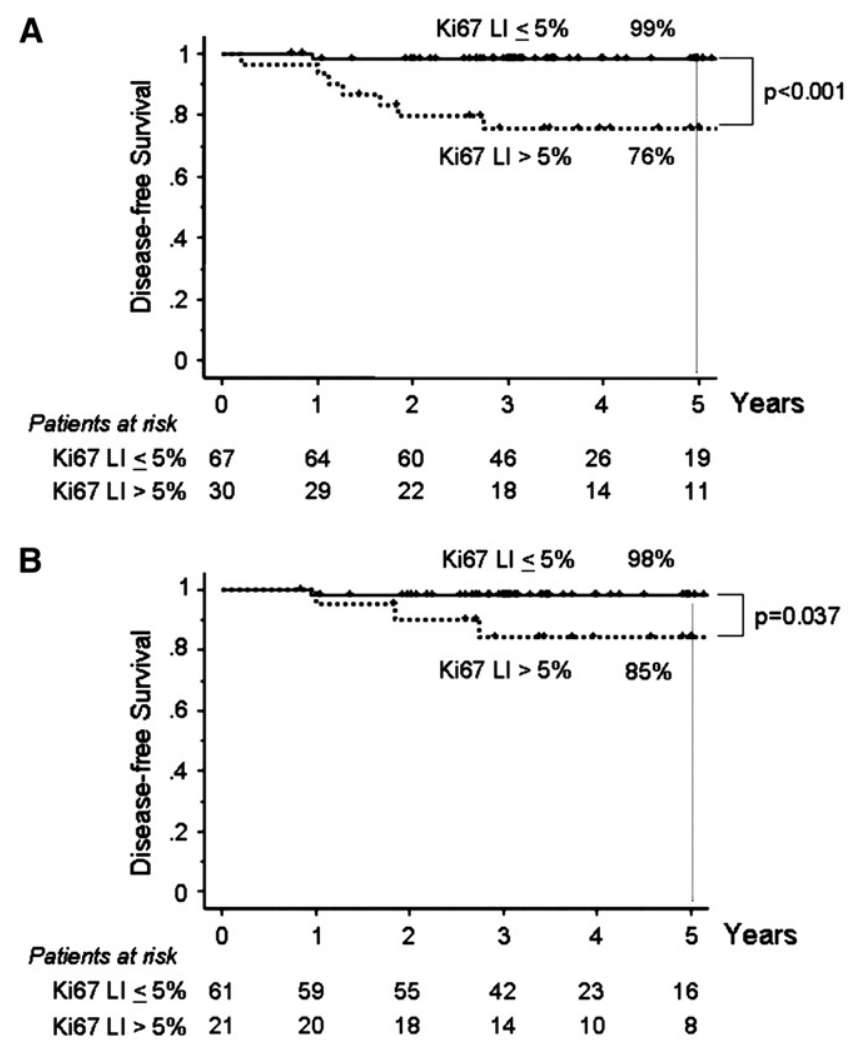

Figure 3. A, Disease-free survival for all patients according to Ki67 labeling index (LI). The Ki-67-high group showed a significantly worse outcome compared with the Ki-67-low group. B, Diseasefree survival for patients without lymph node metastasis according to Ki-67 Ll. The difference in the disease-control ratio was statistically significant.

Ki-67 is generally known as a nuclear protein that is expressed on various cells in other than the G0 phase of the cell cycle. A significant correlation between Ki-67 expression and lymph node metastasis was previously reported for other malignancies, ${ }^{14,15}$ and the prognostic significance of Ki-67 expression for disease-free survival was shown in cases of resected non-small cell lung cancer. ${ }^{16}$ As for Ki-67 expression in early lung cancer, dysplasia and

TABLE 3. Relationship between carcinoembryonic antigen expression in tumor and preoperative serum carcinoembryonic antigen level

\begin{tabular}{lcc}
\hline & IHC $(++)$ & IHC $(-)$ to $(+)$ \\
\hline Serum increased CEA level & 13 & 11 \\
Serum normal CEA level & 9 & 64
\end{tabular}

The cutoff level is less than $5 \mathrm{ng} / \mathrm{mL} . P<.001$, Fisher exact test. $I H C$, Immunohistochemistry for tumor; $C E A$, carcinoembryonic antigen. 

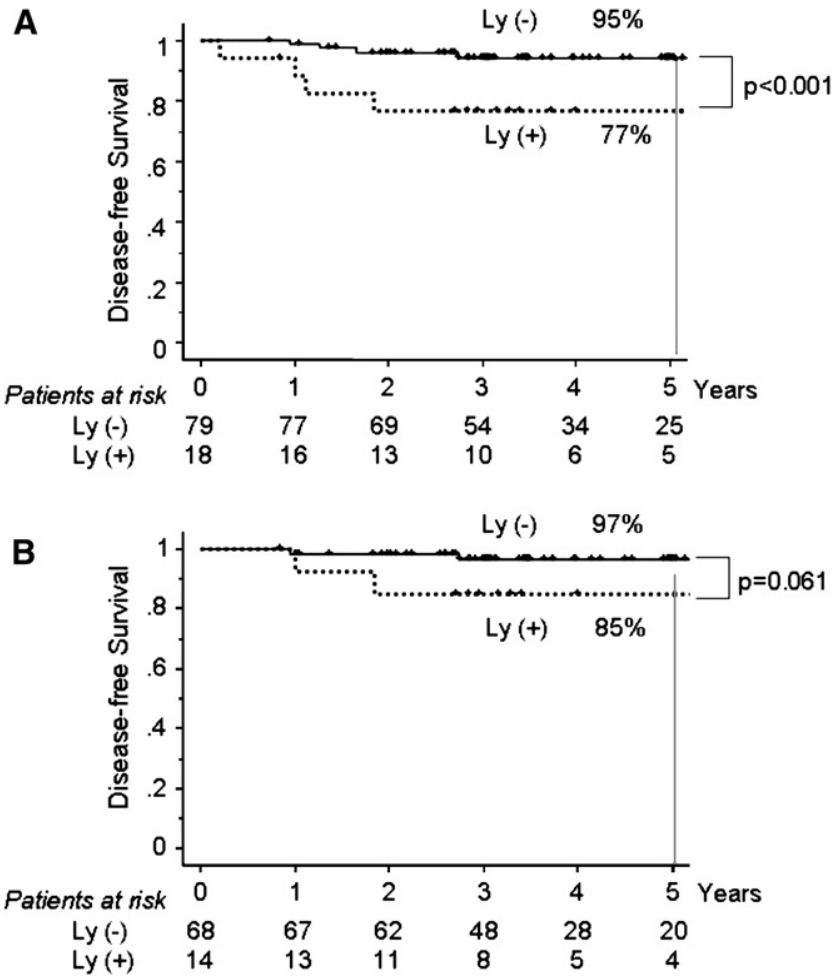

Figure 4. A, Disease-free survival for all patients according to lymphatic invasion (Ly) shown by D2-40 immunohistochemistry results. The Ly $(+)$ group had a significantly worse outcome compared with the Ly (-) group. B, Disease-free survival for patients with pIA disease without lymph node metastasis according to Ly. Disease-free survival for the Ly $(-)$ group tended to be better.

carcinoma in situ, which are precursor lesions for squamous cell carcinoma, and atypical adenomatous hyperplasia have been analyzed, ${ }^{17,18}$ although prognostic significance was not identified in those studies. The present results revealed that a higher Ki-67 LI in a small-sized adenocarcinoma was a clinical predictor for both nodal involvement and postoperative recurrence. We also found that Ki-67 LI was a useful predictor for recurrence in our analysis of patients with pIA disease. Thus an immunohistochemical evaluation of Ki-67 by using preoperative biopsy materials or resected specimens would be useful in clinical practice.

We previously reported that the preoperative serum CEA level can predict nodal involvement in small-sized non-small cell lung cancer lesions smaller than $2 \mathrm{~cm} .{ }^{19}$ Sawabata and coworkers $^{20}$ also noted that lymph node metastasis was found in $23 \%$ of cases of cI non-small cell lung cancer. Therefore we examined CEA expression in tumors and levels in serum to identify the utility of CEA measurement as a predictor for advanced disease in patients with a small-sized pulmonary lesion. However, local CEA expression levels in the
TABLE 4. Multivariate analysis of clinicopathologic factors for postoperative recurrence in patients with resected adenocarcinoma of $2 \mathrm{~cm}$ or less in diameter

\begin{tabular}{lccc}
\hline \multicolumn{1}{c}{ Variables } & Hazard ratio & 95\% CL & P value \\
\hline Microscopic necrosis & & & \\
$\quad$ Negative & 1.000 & & \\
$\quad$ Positive & 2.462 & $0.596-10.167$ & .2130 \\
Ki-67 labeling index & & & \\
$\quad<5 \%$ & 1.000 & & \\
$\quad>5 \%$ & 3.985 & $0.708-22.440$ & .1169 \\
Lymphatic invasion detected & & & \\
$\quad$ with D2-40 antibody & & & \\
$\quad$ Negative & 1.000 & & \\
$\quad$ Positive & 4.767 & $1.069-21.264$ & .0406 \\
Preoperative serum CEA level & & & \\
$\quad$ WNL & 1.000 & & .1094 \\
$\quad>5$ ng/mL & 1.422 & $0.329-6.136$ & .6373 \\
p Stage & & & \\
IA-IB & 1.000 & & .2718 \\
IIA-IIB & 2.660 & $0.465-15.233$ & \\
IIIA & 6.781 & $1.059-43.425$ & .0433 \\
\hline
\end{tabular}

$C L$, Confidence limit; CEA, carcinoembryonic antigen (cutoff value is $5 \mathrm{ng} / \mathrm{mL}$ ); WNL, within normal limit.

tumor had no relationship with nodal metastasis or diseasefree survival in the present cases, although it was reported in another study that all patients with cI disease with an adenocarcinoma who showed a diffuse expression of CEA had a poor prognosis and a lower CEA normalization ratio after surgical intervention. ${ }^{21}$ We consider that measurement of serum CEA levels is a more convenient and useful strategy than immunohistochemical analysis of CEA levels in the tumor for practical management of patients with a smallsized adenocarcinoma.

The D2-40 antibody has been recently reported to be more sensitive for the detection of lymphatic invasion compared with conventional hematoxylin and eosin staining in several types of malignancy, such as gastric, breast, colorectal, and cervical carcinomas. ${ }^{22-25}$ Although some of those previous studies revealed a relationship between lymphatic invasion detected with D2-40 staining and lymph node metastasis, ${ }^{22-24}$ we found no significant relationship in the present study. Nevertheless, lymphatic invasion detected with D2-40 staining had an independent prognostic effect in our cases of small-sized pulmonary adenocarcinoma. We propose that D2-40 staining, which is reliable for evaluation of lymphatic invasion, is useful to predict postoperative relapse in patients who have undergone resection for a small-sized adenocarcinoma.

Although we identified several pathologic predictors for disease relapse in the present study, the preoperative availability of these useful factors has not been established, which is the main limitation of this study. Diagnostic imaging, such 
as positron emission tomography-CT, can provide information regarding oncologic behavior before surgical intervention. ${ }^{26}$ In addition, gene profiling with DNA-chip technology might be another methodology to predict the outcome of patients with resected malignancy. Additional studies of preoperative diagnosis are required to elucidate the means to select small-sized adenocarcinomas with malignant potential.

In summary, histologic findings for microscopic necrosis, Ki-67 LI, CEA expression, and lymphatic invasion, in addition to serum CEA level, should be considered for practical management of patients with a small-sized adenocarcinoma of the lung.

We thank Ms Megumi Sugano, Ms Keiko Fujikawa, Ms Takako Sawamura, Ms Aya Kakuta, and Ms Ayami Saga for their expert technical assistance with the immunohistochemistry examinations. We also thank Ms Naoko Araki for secretarial assistance.

\section{References}

1. Suzuki K, Kusumoto M, Watanabe S, Tsuchiya R, Asamura H. Radiologic classification of small adenocarcinoma of the lung: radiologicpathologic correlation and its prognostic impact. Ann Thorac Surg. 2006;81:413-9.

2. Okada M, Nishio W, Sakamoto T, Uchino K, Hanioka K, Ohbayashi C, et al. Correlation between computed tomographic findings, bronchioloalveolar carcinoma component, and biologic behavior of small-sized lung adenocarcinomas. J Thorac Cardiovasc Surg. 2004;127:857-61.

3. Noguchi M, Morikawa A, Kawasaki M, Matsuno Y, Yamada T, Hirohashi S, et al. Small adenocarcinoma of the lung. Histologic characteristics and prognosis. Cancer. 1995;75:2844-52.

4. Yoshida J, Nagai K, Yokose T, Nishimura M, Kakinuma R, Ohmatsu H, et al. Limited resection trial for pulmonary ground-glass opacity nodules: fifty-case experience. J Thorac Cardiovasc Surg. 2005;129:991-6.

5. Kishi K, Homma S, Kurosaki A, Motoi N, Kohno T, Nakata K, et al. Small lung tumors with the size of $1 \mathrm{~cm}$ or less in diameter: clinical, radiological, and histopathological characteristics. Lung Cancer. 2004;44: 43-51.

6. Koike T, Yamato Y, Yoshiya K, Shimoyama T, Suzuki R. Intentional limited pulmonary resection for peripheral T1 N0 M0 small-sized lung cancer. J Thorac Cardiovasc Surg. 2003;125:924-8.

7. Yoshikawa K, Tsubota N, Kodama K, Ayabe H, Taki T, Mori T. Prospective study of extended segmentectomy for small lung tumors: the final report. Ann Thorac Surg. 2002;73:1055-8.

8. Flieder DB, Port JL, Korst RJ, Christos PJ, Levin MA, Becker DE, et al. Tumor size is a determinant of stage distribution in $\mathrm{t} 1$ non-small cell lung cancer. Chest. 2005;128:2304-8.

9. Nonaka M, Kadokura M, Yamamoto S, Kataoka D, Kunimura T, Kushima M, et al. Tumor dimension and prognosis in surgically treated lung cancer: for intentional limited resection. Am J Clin Oncol. 2003;26: 499-503.

10. Asamura H, Suzuki K, Watanabe S, Matsuno Y, Maeshima A, Tsuchiya R. A clinicopathological study of resected subcentimeter lung cancers: a favorable prognosis for ground glass opacity lesions. Ann Thorac Surg. 2003;76:1016-22.
11. Mountain CF. Revisions in the international system for staging lung cancer. Chest. 1997;111:1710-7.

12. Kessler R, Gasser B, Massard G, Roeslin N, Meyer P, Wihlm JM, et al. Blood vessel invasion is a major prognostic factor in resected non-small cell lung cancer. Ann Thorac Surg. 1996;62:1489-93.

13. Swinson DE, Jones JL, Richardson D, Cox G, Edwards JG, O'Byrne KJ. Tumour necrosis is an independent prognostic marker in non-small cell lung cancer: correlation with biological variables. Lung Cancer. 2002; 37:235-40.

14. Ramires M, David L, Leitao D, Seixas M, Sansonetty F, SobrinhoSimoes M. Ki-67 labelling index in gastric carcinomas. An immunohistochemical study using double staining for the evaluation of the proliferative activity of diffuse-type carcinomas. J Pathol. 1997;182:62-7.

15. Liu M, Lawson G, Delos M, Jamart J, Ide C, Coche E, et al. Predictive value of the fraction of cancer cells immunolabeled for proliferating cell nuclear antigen or Ki-67 in biopsies of head and neck carcinomas to identify lymph node metastasis: comparison with clinical and radiologic examinations. Head Neck. 2003;25:280-8.

16. Scagliotti GV, Micela M, Gubetta L, Leonardo E, Cappia S, Borasio P, et al. Prognostic significance of Ki-67 labelling in resected non small cell lung cancer. Eur J Cancer. 1993;29A:363-5.

17. Meert AP, Feoli F, Martin B, Verdebout JM, Mascaux C, Verhest A, et al. Ki-67 expression in bronchial preneoplastic lesions and carcinoma in situ defined according to the new 1999 WHO/IASLC criteria: a preliminary study. Histopathology. 2004;44:47-53.

18. Kerr KM, Carey FA, King G, Lamb D. Atypical alveolar hyperplasia: relationship with pulmonary adenocarcinoma, p53, and c-erbB-2 expression. J Pathol. 1994:174:249-56.

19. Inoue M, Minami M, Shiono H, Sawabata N, Ideguchi K, Okumura M. Clinicopathologic study of resected, peripheral, small-sized, non-small cell lung cancer tumors of $2 \mathrm{~cm}$ or less in diameter: pleural invasion and increase of serum carcinoembryonic antigen level as predictors of nodal involvement. J Thorac Cardiovasc Surg. 2006;131:988-93.

20. Sawabata N, Ohta M, Takeda S, Hirano H, Okumura Y, Asada H, et al. Serum carcinoembryonic antigen level in surgically resected clinical stage I patients with non-small cell lung cancer. Ann Thorac Surg. 2002;74:174-9.

21. Sawabata N, Hirano H, Inoue M, Okumura Y, Asada H, Takeda S, et al. Immunohistochemical analysis of resected clinical stage I pulmonary adenocarcinomas with high preoperative levels of serum carcinoembryonic antigen. Ann Thorac Surg. 2003;76:203-7.

22. Yonemura Y, Endou Y, Tabuchi K, Kawamura T, Yun HY, Kameya T, et al. Evaluation of lymphatic invasion in primary gastric cancer by a new monoclonal antibody, D2-40. Hum Pathol. 2006;37:1193-9.

23. Van den Eynden GG, Van der Auwera I, Van Laere SJ, Colpaert CG, van Dam P, Dirix LY, et al. Distinguishing blood and lymph vessel invasion in breast cancer: a prospective immunohistochemical study. $\mathrm{Br} J \mathrm{Can}$ cer. 2006;94:1643-9.

24. Walgenbach-Bruenagel G, Tolba RH, Varnai AD, Bollmann M, Hirner A, Walgenbach KJ. Detection of lymphatic invasion in early stage primary colorectal cancer with the monoclonal antibody D2-40. Eur Surg Res. 2006;38:438-44.

25. Urabe A, Matsumoto T, Kimura M, Sonoue H, Kinoshita K. Grading system of lymphatic invasion according to D2-40 immunostaining is useful for the prediction of nodal metastasis in squamous cell carcinoma of the uterine cervix. Histopathology. 2006;49:493-7.

26. Okada M, Tauchi S, Iwanaga K, Mimura T, Kitamura Y, Watanabe H, et al. Associations among bronchioloalveolar carcinoma components, positron emission tomographic and computed tomographic findings, and malignant behavior in small lung adenocarcinomas. $J$ Thorac Cardiovasc Surg. 2007;133:1448-54. 